\title{
Experiencias Desmotivacionales y Motivacionales de Estudiantes Varones de Ingeniería para Estudiar Matemáticas. El Caso de la Universidad Andrés Bello en Santiago de Chile
}

\author{
Emilio J. Castro(1)* e Isaías Miranda ${ }^{(2)}$ \\ (1) Universidad Católica Silva Henríquez, Escuela de Educación Matemática e Informática Educativa, General Jofré 462, \\ Santiago, Chile. (e-mail: ecastro@ucsh.cl) \\ (2) Instituto Politécnico Nacional. CICATA-Legaria. Calzada Legaria 694 Colonia Irrigación 11500, Ciudad de México, \\ Mexico (e-mail: imirandav@ipn.mx) \\ ${ }^{*}$ Autor a quien debe ser dirigida la correspondencia
}

Recibido Abr. 9, 2019; Aceptado May. 31, 2019; Versión final Jul. 21, 2019, Publicado Dic. 2019

\begin{abstract}
Resumen
Con base en la teoría de la autodeterminación y la meta-teoría del dominio afectivo, el objetivo de este artículo es analizar las motivaciones y desmotivaciones de estudiantes de ingeniería para estudiar matemáticas. La metodología utilizada fue la conformación de un grupo focal de ocho estudiantes varones (19 a 24 años), de la Universidad Andrés Bello en Santiago de Chile, a quienes se les preguntó cuáles fueron sus experiencias motivantes (y no motivantes) que contribuyeron en su decisión de estudiar (o no) matemática, y sus motivos específicos de aprender conceptos matemáticos y de responder problemas. Los resultados indican que los estudiantes se motivan o desmotivan por los siguientes temas: "ser como el profesor", "rol del profesor", "evaluaciones", "ser ingeniero" y "rendimiento". Se concluye que estos temas se relacionan con motivaciones extrínsecas. Esto significa que los estudiantes se motivan a estudiar matemática si satisfacen sus necesidades debido a factores externos (relación social, competencia y autonomía) o se desmotivan al no poder satisfacer esas necesidades.
\end{abstract}

Palabras clave: motivación extrínseca; motivación intrínseca; desmotivación; teoría de la autodeterminación; teoría del dominio afectivo; grupo focal

\section{Demotivational and Motivational Experiences of Male Engineering Students to Study Math. The Case of Andrés Bello University in Santiago de Chile}

\begin{abstract}
Based on the self-determination theory and the meta-theory of affective domain, the aim of this article is to analyze the motivations and demotivations of engineering students to study mathematics. The methodology used was the formation of a focus group of eight male students (19 to 24 years of age) from the Andrés Bello University in Santiago-Chile, who were asked about their motivating and non-motivating experience that contributed to their decision to study (or not) mathematics, and their specific motives for learning mathematical concepts and solving to problems. Results show that students are motivated and demotivated by the following themes: "being a teacher", "role of the teacher", "evaluations", "being an engineer" and "performance". It is concluded that motivations are exclusively extrinsic. This means that students are motivated to study mathematics if they satisfy their needs due to external factors (social relations, competence and autonomy) or are discouraged when they cannot satisfy those needs.
\end{abstract}

Keywords: extrinsic motivation; intrinsic motivation; demotivation; theory of self-determination; theory of affective domain; focus group 


\section{INTRODUCCIÓN}

Hace más de una década, Hannula (2006) reconocía que el estudio de la motivación en el aprendizaje de las matemáticas no había sido de interés para los educadores matemáticos. McLeod (1992), al hacer una revisión exhaustiva sobre los estudios relacionados con factores afectivos que influyen en el aprendizaje de la matemática, confirma el reconocimiento de Hannula. Como resultado de su revisión, propuso que en el afecto intervienen creencias, actitudes y emociones. Esto derivó en un marco explicativo llamado Dominio Afectivo. En él, la motivación para el aprendizaje de las matemáticas fue un constructo invisible en el sentido de que, para ser activada por el sujeto, la motivación requiere de creencias, actitudes y emociones. Posterior al trabajo de McLeod (1992), DeBellis y Goldin (2006) agregaron los valores como una nueva dimensión dentro del Dominio Afectivo. Según estos investigadores, los valores hacen referencia a lo que está instalado en el estudiante en un nivel cognitivo mayor que aquél en el que se encuentran a las creencias. De esta forma, la motivación continuó en su papel secundario (invisible), lo que la hizo depender de valores, creencias, actitudes y emociones para su activación.

Ahora bien, en las investigaciones actuales sobre educación matemática, la motivación tiene un papel protagónico en el afecto (Schukajlow et al., 2017; Goldin et al., 2016). El propio Hannula (2012), con la elaboración de su propuesta meta-teórica del Dominio Afectivo, señala que la motivación es un campo muy extenso que merece una atención especial. En este mismo orden de ideas, Goldin et al. (2016) consideran a la motivación, junto con las creencias y las emociones, como los elementos principales del afecto. Así pues, actualmente, en las investigaciones sobre el aprendizaje de las matemáticas se incluye a la motivación como una variable que debe ser considerada de manera importante, dentro de los factores afectivos que influyen en el aprendizaje de la matemática.

Para estudiar la motivación es necesario tomar en cuenta varios factores. Uno de ellos, por ejemplo, es el de las emociones positivas. Oriol-Granado et al. (2017) sugieren que la motivación de estudiantes universitarios se relaciona con las emociones positivas (por ejemplo: alegría, esperanza) que ellos experimentan durante las clases. Así, las experiencias emocionales repercuten directamente en el rendimiento escolar de los estudiantes. Otro factor a considerar para estudiar la motivación tiene que ver con el aburrimiento. En su estudio, Schukajlow (2015) reporta que existe una correlación entre resolver un problema verbal de matemáticas y el aburrimiento. Este investigador sugiere que es deseable que los profesores pidan a sus estudiantes resolver problemas que no causen aburrimiento, pues el hecho de sentirse aburrido puede ocasionar un bajo rendimiento.

Un estudio más específico sobre la motivación y su relación con el éxito académico -entendido no solamente como la calificación, sino también como la satisfacción del estudiante con su centro educativo y su percepción de bienestar- fue desarrollado por Gutiérrez y Tomás (2018). Los resultados de esta investigación indican que los estudiantes universitarios motivados son más propensos a tener éxito académico que aquellos que no lo están. Los estudiantes, según este estudio, se sienten motivados por factores contextuales como el hecho de que los profesores propicien la autonomía de sus estudiantes y los hagan sentir capaces de resolver problemas.

A diferencia de las investigaciones mencionadas en el párrafo precedente, en las que la motivación de los estudiantes es analizada en su relación con factores específicos (motivación-emoción positiva, motivaciónaburrimiento y motivación-éxito académico), en este artículo se estudia la motivación y la desmotivación de estudiantes a partir de diversas experiencias vividas durante su trayectoria estudiantil. En particular, se detallan las motivaciones y desmotivaciones de estudiantes de ingeniería para estudiar matemáticas por medio de la exploración de sus experiencias escolares tanto de los niveles previos como del nivel universitario. La pregunta que guía esta investigación es: ¿qué ha motivado a estudiantes de ingeniería a estudiar matemáticas? Para responderla, se propone un marco conceptual que se basa, por un lado, en la teoría de la autodeterminación, desarrollada por Deci y Ryan (2008), y, por otro, en la meta-teoría del dominio afectivo, desarrollado por Hannula (2012). En específico, el marco conceptual propuesto en este artículo relaciona los conceptos de motivación concebidos en estas dos teorías.

\section{MARCO CONCEPTUAL}

De acuerdo con la teoría de la autodeterminación, la motivación de un individuo se relaciona directamente con la satisfacción de sus necesidades psicológicas básicas: de autonomía, de competencia y de relación social (Ryan, 2012). En la necesidad de autonomía, el sujeto necesita sentir que puede realizar la tarea por sí solo y por su propia voluntad: mientras mayor sea la satisfacción de la necesidad de autonomía, mayor será su motivación y viceversa. La necesidad de competencia es satisfecha cuando el sujeto se siente capaz de realizar una tarea; es decir, siente que su capacidad le permite poder abordar adecuadamente una labor. Ante la ausencia de la satisfacción de la necesidad de competencia, la motivación disminuye; por el contrario, 
si se satisface esta competencia, el sujeto se motiva. En cuanto a la necesidad de relación social, ésta tiene que ver con la interacción del sujeto y su entorno social (amigos, padres, compañeros, profesores, entre otros). Las necesidades de relación social se satisfacen en la interacción con otros; si éstas se satisfacen, se produce la motivación (Deci y Ryan, 2000).

La postulación de la existencia de las necesidades psicológicas básicas permite identificar, según Deci y Ryan (2000), dos tipos de motivaciones: intrínseca y extrínseca. Mientras que la motivación extrínseca (regulación externa) surge cuando el estudiante espera o bien un premio o bien no recibir un castigo externo (por ejemplo, "si estudio, seré el mejor de la clase"), la motivación intrínseca (regulación interna) nace cuando el sujeto muestra curiosidad e interés; cuando no necesita de refuerzos externos o premios (por ejemplo, "me gusta estudiar porque me gusta aprender"). De hecho, en la motivación intrínseca, el estudiante tiene un gusto por realizar la actividad y se siente bien al ejecutarla. Puede darse el caso, también, de que en un individuo no exista ni una motivación extrínseca ni una intrínseca. En este caso, en la teoría de la autodeterminación se dice que hay una desmotivación (por ejemplo, cuando un estudiante no siente impulso para aprender matemáticas) o bien porque la valoración que le otorga a la tarea no es lo suficientemente alta como para querer realizarla, o bien porque no se siente capaz de poder abordar la tarea. Así, para un estudiante desmotivado, no tiene sentido emprender ninguna acción, pues el resultado será el fracaso (Ryan y Deci, 2002).

Ahora bien, la motivación extrínseca e intrínseca, así como la desmotivación, no son perdurables. En la teoría de la autodeterminación, la motivación se reconoce como un continuo (Ryan, 2012); es decir, en un mismo individuo pueden identificarse distintos niveles de motivación en diferentes momentos. En su nivel más bajo, existe una ausencia de motivación (desmotivación). Por ejemplo, un estudiante puede sentirse desmotivado por responder una lista de ejercicios de álgebra; sin embargo, puede existir en él una motivación extrínseca si el profesor otorga un puntaje extra a todo estudiante que resuelva correctamente los problemas de la lista. En este caso se podría decir que, desde la desmotivación, el estudiante tuvo un segundo nivel de motivación, ocasionado por la regulación externa. Finalmente, en el nivel motivacional más alto, se tiene a la motivación por regulación interna, es decir, la motivación intrínseca. En el ejemplo del estudiante que resuelve la lista de problemas, la motivación intrínseca podría surgir cuando él se de cuenta que la lista contiene problemas que le son de su interés. Se entiende entonces que el estudiante puede pasar por distintos estados motivacionales sin tener una motivación o desmotivación establecida de forma permanente (Ryan y Deci, 2000). Debe aclararse que el tránsito entre los distintos niveles de motivación no sigue un orden establecido (por ejemplo, del nivel más bajo al más alto o viceversa). Las motivaciones y desmotivaciones ocurren de diversas maneras.

\section{METODOLOGÍA}

Para detallar lo que a estudiantes de ingeniería les ha motivado estudiar matemáticas, se consideró pertinente situar la metodología dentro de un paradigma interpretativo de la investigación. Con este tipo de paradigmas, la atención se centra en el estudio de significados, interpretación y comprensión de las acciones de las personas en su vida social (Daly, 2007). Así, se conformó un grupo focal de 8 estudiantes (19-24 años), todos ellos varones, de la Universidad Andrés Bello en Santiago de Chile, durante los meses de septiembre y octubre de 2016. El motivo de esta conformación se debió a que la clase estuvo conformada únicamente por varones. Vale destacar que los aspectos relacionados con el género, según la investigación a estudiantes de Ingeniería de Sistemas de la Universidad de Cartagena (Colombia) realizada por Acevedo et al. (2015), no tienen un papel importante respecto de la motivación para el aprendizaje. Además, de acuerdo con Soler (1997), las características principales de los grupos focales están relacionadas con la interacción de los participantes y con que éstos se desenvuelvan en concordancia con sus propias necesidades, permitiéndoles que manifiesten experiencias y puntos de vista personales. De esta forma, consideramos que el grupo focal de varones es el adecuado para la toma de datos.

En el momento de realizar la investigación, los estudiantes cursaban la asignatura de cálculo integral. El grupo focal se organizó según características similares, como la de cursar la misma carrera, estar en un mismo nivel educativo, ser del género masculino y tener disposición a colaborar en la investigación (Collins, Onwuegbuzie y Jiao, 2006; Creswell, 2008). El moderador que guió la entrevista del grupo focal fue el primer autor de este artículo, quien también impartió el curso de cálculo integral. Con base en las sugerencias de Cohen et al. (2007), consideramos pertinente recalcar que el doble rol del primer autor (profesor e investigador) le da validez a la investigación, pues su participación fue observacional no participante; es decir, mientras su rol de profesor le servía para generar confianza en los estudiantes y para moderar el tono de sus preguntas, él podía tomar nota de las respuestas de los estudiantes y hacer que de éstas se generaran discusiones al interior del grupo focal. Además, al participar como observante de la dinámica del grupo focal, el profesor tuvo la posibilidad de dar sentido a la tonalidad de las respuestas y de observar el contexto en el que éstas fueron emitidas. De esta forma, la metodología utilizada en esta investigación es cualitativa, en su modalidad de observación participante. A los estudiantes se les pidió que discutieran sobre los temas relativos a la 
motivación que se les propuso en el grupo focal. El grupo de relatos que se extrajo está fielmente rescatado de la discusión generada. El medio para la recolección de información fue la videograbación. La interacción del grupo focal tuvo una duración aproximada de una hora.

El proceso de formulación de las preguntas (cuya duración, de acuerdo con las recomendaciones de Morgan et al. (1998), fue de entre 5 y 10 minutos) elaboradas para el grupo focal comenzó con la clarificación del tema de motivación. Posteriormente, se realizó una lluvia de ideas y se crearon borradores con preguntas. Las preguntas planteadas al grupo focal fueron grabadas en audio y video, previa autorización de cada uno de los participantes (se hizo énfasis en que el video sería únicamente utilizado para esta investigación). A los estudiantes se les solicitó que tomaran en cuenta su experiencia estudiantil en los niveles de enseñanza básico, medio y universitario para contestar las siguientes preguntas: 1) ¿En cuáles de ellos has vivido experiencias que te motiven a estudiar matemáticas?; 2) ¿En cuáles de ellos has vivido experiencias que te desmotiven a estudiar matemáticas?; y 3) ¿Si tuvieses que elegir uno de ellos en cuál te sentiste más motivado y en cuál te sentiste menos motivado?

Para explorar qué activa su motivación, según su propia percepción, a los estudiantes se le plantearon las siguientes preguntas: 1) ¿Qué te motiva o impulsa a asistir a la clase de cálculo integral o ecuaciones diferenciales?; 2) ¿Qué te desmotiva o te detiene a asistir a la clase de cálculo integral o ecuaciones diferenciales?; 3) ¿Qué te motiva o impulsa a resolver problemas?; 4) ¿Qué te desmotiva o te detiene resolver problemas?; y 5) ¿Qué te motiva a persistir a resolver problemas cuando es difícil?

Las transcripciones de las discusiones se analizaron con la técnica del análisis temático en el programa Atlas.ti 8.2.4. Con el análisis temático es posible identificar, organizar y analizar en profundidad las respuestas de los estudiantes. Una lectura sistemática de sus respuestas permite entender y explicar las complejidades de sus motivaciones. El análisis temático (Braun y Clarke, 2006) se compone de un proceso que consta de 6 fases, las cuales son: familiarización de los datos (transcripción, lectura y relectura, anotación de ideas generales), generación de códigos iniciales (codificación de los aspectos más importantes de los datos, recopilación de datos relevantes para cada código), búsqueda de temas (elaboración de un mapa temático de temas), definición y denominación de temas (análisis continuo de cada tema, generación de definiciones y nombres para cada tema) y preparación de informe (selección y análisis final de los fragmentos de textos seleccionados y redacción de un informe académico del análisis).

La validez y confiabilidad se obtuvo con dos mediciones. En la primera medición se realizó un juicio de expertos (Ahuvia, 2001): a 3 expertos se les entregó una parte de las transcripciones (10\%), considerando que esta parte abarcaba todos los temas identificados en las 6 fases del análisis. Junto con los códigos generados correspondientes a esas transcripciones, los expertos evaluaron si las codificaciones efectuadas cumplieron con ser relevantes, significativas, claras y pertinentes (Fereday, 2006). La segunda medición se realizó con el trabajo entre codificadores (Vaismoradi et al., 2013). En ella, un codificador externo a la investigación coincidió en la codificación realizada en la investigación.

\section{RESULTADOS Y DISCUSIÓN}

Del análisis de los resultados se pudo obtener información relevante con respecto a la motivación que mostraron los estudiantes (nombrados como E1, E2, ..., E8) en pasajes que ellos mismos relatan como importantes en sus propias experiencias escolares, tanto de su pasado como de su presente, sobre el aprendizaje de la matemática. Del análisis temático, las respuestas de los estudiantes se agruparon en los siguientes cinco temas: "Ser como el profesor", "Rol del profesor", "Evaluaciones", "Ser ingeniero" y "Rendimiento". A continuación, se describen las características de cada tema y se muestran las respuestas que a éste lo caracterizan.

\section{Ser como el profesor}

El primer tema surgido del análisis fue llamado "Ser como el profesor". Se refiere a estudiantes que relatan haberse sentido motivados o desmotivados en la medida en que sean capaces de explicar contenidos como lo hace el profesor. Por ejemplo, un estudiante, E1, ante la pregunta sobre cuáles niveles de enseñanza han provocado experiencias motivantes, respondió con referencia a la enseñanza básica: "Me pasó casi lo mismo que dijo él [se refiere a su compañero]: por las ecuaciones. Que no me acuerdo bien cuándo [fue la lección de ecuaciones], estoy entre [los 13 y 14 años de edad], yo lo entendí mejor que todos, pude explicar a mis compañeros y fui ayudante del profesor para explicarle a los demás también. Ahí me empezó a gustar el tema de la matemática y quise estudiar algo con matemática". Como se observa en la respuesta anterior, algunos estudiantes se motivan a estudiar cuando pueden aprender alguna temática antes que sus pares y así enseñar esta temática a sus compañeros. En este caso, lo que E1 encuentra motivante es actuar como su profesor. 
Otra respuesta que ilustra la motivación de "Ser como el profesor" con referencia a la enseñanza superior fue: "Yo en lo personal, a mi me gusta aprender cosas nuevas. Eso es lo que más me gusta. Sobre todo, porque después mis compañeros me preguntan y yo les puedo decir: miren así se resuelve el problema, es fácil” (E2). En esta respuesta se observa una motivación extrínseca de satisfacer una relación social: la de tener un lugar importante, como la del profesor, en su entorno social; la de ser una persona necesaria para sus pares. La motivación de E2 está en concordancia con lo desarrollado por Hidi y Renninger (2006), quien señaló que el interés, estrechamente relacionado con la motivación, se puede desencadenar por situaciones sociales o de contenido. En este tipo de situaciones, los estudiantes tienen la oportunidad de regular su proceso epistémico (Bikner-Ahsbahs y Halverscheid, 2014). Además, en E2 se satisface la necesidad de competencia, debido a que él se siente en propiedad de las capacidades necesarias para poder aprender matemática. Este es un hallazgo que coincide con los trabajos de Rakoczy et al. (2013) y de Schukajlow y Krug (2014). Todo lo anterior se decanta entonces en una motivación extrínseca hacia el aprendizaje de la matemática.

Ahora bien, aun cuando los estudiantes declaren experiencias no satisfactorias, se observa que su desmotivación no se debe al contenido matemático, sino a no poder ser como el profesor. Tal es el caso de E1. Este estudiante previamente había afirmado que una experiencia motivante en la enseñanza básica fue la de poder explicar a sus compañeros los contenidos matemáticos; sin embargo, al pedirle que describiera una experiencia desmotivante al estudiar matemáticas, afirmó: "Yo también en la [enseñanza] básica, con geometría, no me gustaba geometría, me iba pésimo. No entendía los ángulos, eso" (E1). En esta respuesta, E1 asegura que no comprendía el contenido relacionado con los ángulos de u figuras geométricas. Si se toma en cuenta la primera respuesta, puede inferirse que esta falta de comprensión no es la causa de su desmotivación para el estudio de las matemáticas, sino la imposibilidad de no poder enseñar el concepto de ángulos a sus compañeros. Dicho de otra manera, el mismo estudiante que mostraba una motivación extrínseca dada por la satisfacción de la necesidad de competencia y relación social ("Ser como el profesor"), ahora muestra, en el caso de la geometría, una desmotivación que se caracteriza por una valoración (que él considera inalcanzable) a la matemática, pero no hacia su deseo de "Ser como el profesor". Este es un hallazgo que está en la misma línea de lo expuesto por Schukajlow (2015), quien señaló que los estudiantes valoran la matemática y les encantaría aprenderla, pero a veces simplemente no se sienten capaces de abordar esta tarea. Otra respuesta a la pregunta relacionada sobre la descripción de una experiencia desmotivante, y de la cual puede inferirse que el factor motivante para estudiar matemáticas es el profesor, es la siguiente: "no sé, cuando pasaban logaritmos y esas cosas (enseñanza media), nunca entendi" (E5). En este caso, E5 asegura no haber entendido los logaritmos, pero la causa de su desmotivación es la no satisfacción de necesidades básicas provocadas en los estudiantes.

\section{Rol del Profesor}

El segundo tema que se detectó es el denominado "Rol del Profesor". Este se refiere al rol que juega el profesor en la motivación de los estudiantes. Un ejemplo de la influencia motivacional del profesor de enseñanza media se observa en la siguiente respuesta: "El tema es que el profesor [hizo] unos comentarios súper desagradables y desmotivadores, a pesar de que era buen profe nos enseñaba la materia y todo, dijo que nosotros no teníamos cabida para postular a carreras tipo A, así como ingeniería y cosas así. Un día llegó [a la clase] enojado y dijo de qué servía que nos pasara las derivadas y cosas así, [en el curso avanzado de matemáticas para secundaria], si no lo íbamos a ocupar" (E6). Como se nota en el relato de E6, el profesor, a través de su discurso, influye en la satisfacción de la necesidad de competencia del estudiante, pues, a su juicio, los estudiantes no son merecedores de conocer ciertos contenidos de "índole superior", como es el caso de las derivadas. Sin embargo, debe considerarse que, pese al comentario del profesor, el estudiante valora la labor del profesor como buena en lo que respecta a impartir su clase. Se puede inferir que el estudiante señala que el profesor tiene dos facetas: la de docente que imparte su clase y la de consejero o sabio (metafóricamente hablando). Es precisamente esta dualidad por la que es valorada su labor.

Otro ejemplo sobre cómo el rol del profesor influye en la motivación de los estudiantes se observa en la respuesta de E4: "Yo, cuando me cambié de colegio, y empezaron a pasar las ecuaciones, me costaba mucho entender al revés de ellos [de sus compañeros]. Todo al revés me costaba entender lo del álgebra, por qué había un $x$, por qué había un y. Me costaba mucho. Mi profesor me decía que era tonto y eso me desmotivaba mucho. Pero después, ya en la media, en tercero o cuarto medio me empezó a ir súper y tenía el mejor promedio en matemáticas, no como ahora en la universidad" (E4). En este caso el profesor, con base en el desempeño del estudiante, emite un juicio hacia él: le dice tonto. El estudiante señala que se desmotiva debido a que espera un refuerzo afectivo de parte del profesor, pero por el contrario recibe el castigo de sus calificativos. Vale mencionar que, pese a las palabras de los profesores, los estudiantes terminaron estudiando ingeniería. De hecho, mostraron, como en el caso de estudiante $\mathrm{E} 4$, una mejoría en su rendimiento en los años posteriores. Hay que considerar que estos estudiantes tienen una alta resiliencia, por lo que el profesor no fue un hito que marcó profundamente su vida académica. En este escenario, el estudiante se mueve hacia una desmotivación en la que no se sienta capaz de abordar la tarea debido a esta mezcla entre 
no logro de apropiación de los contenidos y, o bien el discurso del profesor, o bien una motivación extrínseca en su grado mínimo. Sin embargo, el estudiante resiste el embate afectivo y persiste en el aprendizaje de la matemática.

El "Rol del Profesor", como tema de motivación de los estudiantes, también puede tener una connotación positiva. Cuando a los estudiantes se les consultó acerca de por qué asisten a clases, señalaron que la clase es un apoyo para clarificar las ideas y conceptos. E7 lo aclara de la siguiente manera en el contexto de la enseñanza superior: "En la clase es todo lo contrario, es clarificador. Porque uno mira algo y esto, ¿cómo se hace? y después, cuando explica uno, va entendiendo que son pasos que hay que seguir". Para E7, entonces, la motivación extrínseca para aprender matemáticas consiste en que la clase es un espacio en el que él puede entender lo que no ha entendido. Los trabajos de Frenzel et al. (2009) y de Bieg et al. (2017) apuntan en este mismo sentido. En el primero se observa que el entusiasmo que el profesor muestra en sus clases tiene un efecto positivo en los estudiantes, lo que coincide con lo señalado por Farias y Pérez (2010) y por Lobato y Madinabeitia (2011), mientras que en el segundo se asegura que los métodos de enseñanza implementados por los profesores tienen un efecto en las emociones de los estudiantes. La respuesta de E7 expresa la existencia de una motivación ocasionada por el hecho de sentirse competente para entender conceptos matemáticos durante la clase. Al parecer, como lo señala Nguyen (2011), las explicaciones del profesor sobre los significados de los conceptos matemáticos durante las clases pueden restaurar la confianza del estudiante a pesar de que éste haya tenido experiencias desmotivadoras.

\section{Las evaluaciones}

El tercer tema identificado en la motivación de los estudiantes se refiere a "las evaluaciones". Al pedirle que señale en qué nivel educativo tuvo experiencias cuyos efectos fueron desmotivantes para él, E3 responde en el contexto del nivel universitario: "Yo a veces digo: a lo mejor no sirvo para la matemática. Es en el momento en que uno está en la prueba". Como puede observarse, la experiencia que E3 evoca en su respuesta está relacionada con la evaluación del curso. Es en el momento en que debe resolver una prueba en el que E3 se desmotiva. En la prueba, el estudiante se cuestiona sobre su competencia con respecto al aprendizaje de toda la matemática. Sobre esta desmotivación en el mismo contexto universitario, un estudiante dijo: "Si, ese es el momento [se refiere a la evaluación], cuando quedas en blanco [no sabes qué responder]" (E8). La tensión de ser evaluado mueve emocionalmente a los estudiantes brevemente hacia la desmotivación; son los momentos de incertidumbre hacia el resultado que tendrá la evaluación. Esto se debe a que el resultado de una evaluación tiene gran valor tanto para el estudiante como para todo su entorno (por ejemplo, padres, sus profesores, la institución donde estudia, sus compañeros). Al igual que en el caso de la motivación relacionada con "Ser como el profesor", el estudiante de ingeniería resiste al embate afectivo que significa la evaluación, por lo que se encuentra en un estado inestable de motivación extrínseca, debido a que su necesidad de competencia está permanentemente a prueba en el periodo universitario.

Las experiencias motivacionales generadas por la capacidad de resolver problemas que siente el estudiante dependen del momento en el que se encuentra la clase. Esta aseveración la ilustra la respuesta de E3, quien, al preguntarle si se siente desmotivado en las clases de ecuaciones diferenciales, responde: "No, no pasa eso. Es en el momento en que uno está en la prueba y dice [expresa una grosería]..." (E3). Como se puede observar, E3 no se siente desmotivado en el momento de las clases, sino en el de la evaluación (prueba). De acuerdo con Ryan (2012), puede decirse que la motivación es continua. Más claramente, para E3 la clase de ecuaciones diferenciales le provoca motivación (en este caso, extrínseca); sin embargo, la prueba, un momento en el cual él sabe que sus conocimientos serán evaluados, su motivación desaparece. Al parecer, la prueba es un momento de tanta tensión emocional para E3 que, incluso, lo caracteriza con una grosería. Ahora bien, la motivación para aprender matemáticas durante la clase y la desmotivación para ese mismo aprendizaje durante la evaluación están relacionadas con su necesidad de sentirse competente para comprender conceptos matemáticos. En las clases, al sentirse confiado de que sus dudas serán resueltas, su motivación de seguir aprendiendo refuerza su sentimiento de sentirse competente; sin embargo, como en la prueba no hay la oportunidad de que sus dudas sean resueltas, o bien por sus compañeros, o bien por el profesor, su sentimiento de incompetencia se presenta.

\section{Ser ingeniero}

El cuarto tema detectado en el análisis que motiva a los estudiantes a estudiar matemáticas es "ser ingeniero". Esta motivación se refiere a lo que los estudiantes sienten y relacionan con el hecho de ser ingenieros. Con respecto a las preguntas que indagan sobre lo que activan los estudiantes para estudiar ingeniería, una de las respuestas con respecto al nivel superior fue: "[Lo que me motiva es] Poder adquirir conocimiento, ya que, igual me cuesta un poco entonces, siento que es como un desafío más allá de pasar el ramo [aprobar el curso] o decir me voy a atrasar, eso motiva harto pasar el ramo y terminar con la carrera, eso es importante" (E4). Como lo indica esta respuesta, aprobar los cursos es una tarea fundamental para lograr el fin relacionados 
con una motivación extrínseca general: ser ingeniero. La matemática se vuelve entonces una barrera más que superar en la carrera por ser ingeniero, lo que denota claramente una motivación extrínseca. Así. aprobar el curso de matemáticas será una muestra de competencia y de autonomía hacia la meta final.

La motivación de ser ingeniero se refleja, también, en la respuesta de E8: "En la [educación secundaria], me fue bien en matemáticas. Ahí fue cuando decidí estudiar ingeniería. Fue como en tercero medio, y ahí cuando más motivado me senti” (E8). De este último extracto se infiere que el hecho de saber matemáticas está asociado con la carrera de ingeniería y, consecuentemente, el estudiante que tiene un buen desempeño en esta materia se sentirá motivado. Ahora bien, esta motivación no es necesariamente intrínseca. Como lo indica la respuesta de E4, la matemática es un medio para ser ingeniero. En este sentido, la motivación de estudiarlas es extrínseca.

En la siguiente respuesta se ilustra aún más la motivación que tienen los estudiantes de estudiar matemáticas por el hecho de que éstas son un medio para convertirse en ingenieros. "Para ser ingeniero, cómo un ingeniero va a decir [que no sabe matemática]. No, si yo no sé matemática. Esta carrera es si yo soy ingeniero es porque soy hábil en derivadas, en integrales; sé manejar el asunto al revés y al derecho" (E7). En esta respuesta puede observarse la asociación explícita que hace el estudiante entre la capacidad para aprender matemática con el hecho de ser ingeniero. Los estudiantes se sienten motivados para ser ingenieros por el hecho de saber que su desempeño en las clases de matemáticas es positivo. Las respuestas de estos estudiantes están en concordancia con los hallazgos de Matthews et al. (2013), quienes sostienen que la alta motivación (extrínseca) de los estudiantes de ingeniería de su estudio está relacionada con el desempeño de ellos en un curso específico de Cálculo.

\section{Rendimiento}

El tema "Rendimiento" considera la influencia motivacional del promedio de notas. Vale aclarar que la diferencia de este tema con el de "Evaluaciones" es que, en aquél, se considera la calificación en sí misma, mientras que en éste se toma en cuenta el momento o situación específicos en los que se emitió la evaluación. La motivación relacionada con el rendimiento puede tener distintos matices. En la respuesta de E4, analizada previamente en el tema "Rol del profesor", se observa cómo el rendimiento que él tuvo en la enseñanza media lo motivó a estudiar matemáticas (... Mi profesor me decía que era tonto y eso me desmotivaba mucho, pero después ya en la media en tercero cuarto medio me empezó a ir súper y tenía el mejor promedio en matemáticas. Pero esta motivación contrasta con su desmotivación en la universidad (... [continuación de la respuesta anterior] No como ahora en la universidad), pues E4 es consciente de que el rendimiento en sus estudios de ingeniería no es tan satisfactorio como en la enseñanza media. Con esta desmotivación, relacionada con un bajo rendimiento, caracterizado por la obtención de bajas calificaciones, la necesidad de E4 de sentirse competente para aprender matemáticas no está satisfecha. Esta sensación de incompetencia puede, peligrosamente, llevar al estudiante a una desmotivación más profunda que la presenta durante el curso.

En resumen, las distintas experiencias motivacionales de los estudiantes se pueden observar en la tabla 1:

Tabla 1: Temas motivacionales identificados en las respuestas de los estudiantes en distintos niveles educativos.

\begin{tabular}{|c|c|c|c|}
\hline Nivel Educativo & Desmotivación & Motivación Extrínseca & Motivación Intrínseca \\
\hline Enseñanza Básica (Primaria) & No hubo & Ser como el profesor & No hubo \\
\hline Enseñanza Media (Secundaria) & $\begin{array}{l}\text { Rol del Profesor } \\
\text { Rendimiento } \\
\text { Ser como el profesor }\end{array}$ & $\begin{array}{l}\text { Ser ingeniero } \\
\text { Ser como el profesor } \\
\text { Rendimiento }\end{array}$ & No hubo \\
\hline Enseñanza Universitaria & $\begin{array}{l}\text { Evaluaciones } \\
\text { Rendimiento } \\
\text { Ser como el profesor }\end{array}$ & $\begin{array}{l}\text { Ser Ingeniero } \\
\text { Rol del Profesor }\end{array}$ & No hubo \\
\hline
\end{tabular}

Como se observa en la tabla 1, los estudiantes no manifestaron experimentar una motivación intrínseca en el estudio de las matemáticas en ninguno de los niveles educativos. En los estudiantes entrevistados, la desmotivación para estudiar matemáticas se presenta a partir de la enseñanza media. El tema recurrente asociado a esta desmotivación es el Rendimiento. De hecho, un bajo rendimiento, asociado a calificaciones insuficientes, puede originar un sentimiento de incompetencia en los estudiantes; es decir, puede hacer sentir a los estudiantes poco o nada hábiles para responder ejercicios. Es interesante notar que la desmotivación en la enseñanza media se asocie con el "Rol del Profesor", pero que éste sea, por el contrario, una motivación extrínseca para los estudiantes de ingeniería. 
Una experiencia motivacional extrínseca presente tanto en la enseñanza media como en la universitaria es el de "Ser ingeniero". Parece que los deseos de los estudiantes de convertirse en ingenieros ejercen una acción netamente positiva en ellos. Este tema corresponde a las aspiracionales, muy humanas, de "querer ser como alguien". De la misma manera, la figura del profesor es importante como motivación externa. En la tabla 1 se puede observar que, por un lado, durante la enseñanza media, la figura del profesor desmotiva a los estudiantes a estudiar matemáticas; por otro, en la enseñanza universitaria, el desempeño del profesor es el factor que motiva. Así, lo motivante de estos dos temas, "Ser como el profesor" y "Rol del profesor", depende de su influencia en los estudiantes. Por ejemplo, en el caso del tema "Rol del profesor", el profesor, a través de su discurso, puede apoyar o minar la necesidad de los estudiantes de sentirse competentes para aprender matemáticas; una necesidad que, además, les otorga autonomía de pensamiento y acción en el momento de resolver problemas. En el caso del tema "Ser como el profesor", las explicaciones que sean significativas para los estudiantes pueden no solo favorecer una mejor comprensión conceptual, sino generar un sentimiento de seguridad para resolver problemas desafiantes.

En la tabla 1 se puede también observar que el "Rendimiento" desmotivó a los estudiantes tanto en su enseñanza media como en su enseñanza universitaria. Tener un bajo rendimiento, comúnmente relacionado con la obtención de calificaciones no aprobatorias, afecta a la necesidad de los estudiantes de sentirse competentes para estudiar matemáticas. Sin embargo, esta afectación es de corta duración, pues sentirse poco o nada competente para aprender matemáticas puede revertirse relativamente rápido si en otro momento de la clase el estudiante siente que su rendimiento ha sido satisfactorio. En otras palabras, la desmotivación debida al Rendimiento tiene estrecha relación con el desempeño, positivo o negativo, de los estudiantes.

Finalmente, se revela que una de las limitaciones resultantes de la metodología del grupo focal es la de no disponer de un panorama más general de las motivaciones de todos los estudiantes del grupo participante del estudio. Por otro lado, el hecho de que los estudiantes participantes de esta investigación no hayan manifestado tener motivaciones intrínsecas para estudiar matemáticas, siendo la matemática una de las disciplinas más importantes en las carreras de ingeniería, los estudiantes desean estudiarla en función de factores externos, lo que da pie a consideraciones importantes sobre los motivos reales de los estudiantes de ingeniería para estudiar matemática.

\section{CONCLUSIONES}

Desde el punto de vista metodológico, la característica principal de este estudio fue la de indagar momentos importantes de la vida escolar de los estudiantes en los que su motivación (o desmotivación) por estudiar matemáticas se hizo evidente. Con base en el análisis de los datos, argumentamos que no hay indicios de que ellos hayan estudiado matemáticas debido a motivaciones intrínsecas; es decir, los estudiantes no manifestaron haber estudiado matemáticas por gusto personal. De hecho, los resultados indican que estas motivaciones son exclusivamente de tipo extrínsecas.

De las respuestas de los estudiantes se identificaron temas motivacionales específicos de los estudiantes de como el de "Ser ingeniero" y "Ser como el profesor". Otros temas motivacionales dependen principalmente del desempeño de los estudiantes. Por ejemplo, el "Rendimiento" y las "Evaluaciones" son motivantes para estudiar matemáticas si los estudiantes consideran que tanto su rendimiento como las evaluaciones hechas por el profesor respecto de su desempeño en el aula han sido positivos; por el contrario, estos temas pueden ser desmotivantes para el aprendizaje de las matemáticas si los estudiantes los consideran negativos. El tema "Rol del profesor" surge de la influencia motivacional que genera el actuar del profesor en el aula, el cual puede ser motivador o desmotivador, destacando que el profesor puede ayudar a retomar la confianza y motivación en estudiantes que estén desmotivados, el profesor influye motivacionalmente con su disposición a la clase y que éste puede marcar a los estudiantes a futuro.

En los estudiantes también se identifican dos necesidades que requieren ser satisfechas para que los estudiantes se sientan motivados. Los estudiantes desean, por un lado, sentirse capaces de comprender contenidos matemáticos y, por otro, saberse reconocidos por sus pares 0 , incluso, por sus profesores. Desde el punto de vista afectivo, satisfacer estas necesidades no solo contribuye a que los estudiantes estén motivados a estudiar matemáticas, sino también a valorar positivamente su sentido de pertenencia a un grupo de personas que se dedican a lo que ellos han imaginado o deseado ser. Dicho de otra manera, al querer "ser ingenieros" o "ser como el profesor", los estudiantes reconocen que una de las características específicas de "ser un ingeniero" es la de entender el significado de los conceptos matemáticos. Es este entendimiento el que, a su vez, permite que la necesidad de sentirse reconocido requiera ser cumplida con la intención de tener motivación para estudiar. 


\section{REFERENCIAS}

Ahuvia, A., Traditional, interpretive, and reception based content analyses: Improving the ability of content analysis to address issues of pragmatic and theoretical concern, doi: 10.1023/A:1011087813505, Social indicators research, 54(2), 139-172 (2001)

Acevedo, D., J.D. Torres y D.F. Tirado., Análisis de los Hábitos de Estudio y Motivación para el Aprendizaje a Distancia en Alumnos de Ingeniería de Sistemas de la Universidad de Cartagena (Colombia), doi: 10.4067/S071850062015000500007, Formación Universitaria, 8(5), 59-66 (2015)

Bieg, M., T. Goetz y otros cinco autores, Teaching methods and their impact on students' emotions in mathematics: An experience-sampling approach, doi:10.1007/ s11858-017-0840-1, ZDM Mathematics Education, 49(3), $307-322$ (2017)

Bikner-Ahsbahs, A. y S. Halverscheid, Introduction to the theory of interest-dense situations (IDS), doi: 10.1007/978-3319-05389-9_7, Networking of theories as a research practice in mathematics education, pp 97-113, Springer, Berlin, Alemania (2014)

Braun, V. y V. Clarke, Using thematic analysis in psychology, doi: 10.1191/1478088706qp063oa, Qualitative Research in Psychology, 3(2), 77-101 (2006)

Cohen, L., L. Manion y K. Morrison, Research Methods in Education, 6a Ed., pp 5-591, Routledge, New York, EE.UU (2007).

Collins, K. M., A. J. Onwuegbuzie y Q. G. Jiao, Prevalence of mixed-methods sampling designs in social science research, doi: 10.2167/eri421.0, Evaluation and Research in Education, 19(2), 83-101 (2006)

Creswell, J.W., Qualitative inquiry and research design: choosing among five traditions, 1a Ed., Sage Publications, Thousand Oaks, California (1998)

Daly, K.J., Qualitative methods for family studies and human development, 1ㄹ Ed., Sage Publications, Thousand Oaks, California (2007)

DeBellis, V.A. y G.A. Goldin, Affect and meta-affect in mathematical problem solving: A representational perspective, doi: 10.1007/s10649-006-9026-4, Educational Studies in Mathematics, 63(2), 131-147 (2006)

Deci, E.L. y R.M. Ryan, The "What" and "Why" of goal pursuits: Human needs and the selfdetermination of behavior, doi: 10.1207/S15327965PLI1104, Psychological Inquiry, 11(4), 227-268 (2000)

Deci, E.L. y R.M. Ryan, Facilitating optimal motivation and psychological well-being across life's domains, doi: 10.1037/0708-5591.49.3.262, Canadian Psychology, 49(1), 14-23 (2008)

Farías, D. y J. Pérez, Motivación en la Enseñanza de las Matemáticas y la Administración, doi: 10.4067/S071850062010000600005, Formación Universitaria, 3(6), 33-40 (2010)

Fereday, J., Demostrating rigor using themathic analysis: A hybrid approach of inductive and deductive coding and theme development, doi: 10.1177/160940690600500107, International Journal of Qualitative Methods, 5(1), 80-92 (2006)

Frenzel, A.C., T. Goetz y otros tres autores, Emotional transmission in the classroom: Exploring the relationship between teacher and student enjoyment, doi: 10.1037/a0014695, Journal of Educational Psychology, 101(3), 705-716 (2009)

Goldin, G., M. Hannula y otros nueve autores, Attitudes, Beliefs, Motivation, and Identity in Mathematics Education: An Overview of the Field and Future Directions, doi:10.1007/978-3-319-32811-9_1, Springer Open, 1-35 (2016)

Gutiérrez, M. y J.M. Tomás, Clima motivacional en clase, motivación y éxito académico en estudiantes universitarios, doi: 10.1016/j.psicod.2018.02.001, Revista de Psicodidáctica, 23(1), 94-101 (2018)

Hannula, M.S., Motivation in Mathematics: Goals Reflected in Emotions, doi:10.1007/s10649-005-9019-8, Educational Studies in Mathematics, 63(2), 165-178 (2006)

Hannula, M.S., Exploring new dimensions of mathematics-related affect: embodied and social theories, doi:10.1080/14794802.2012.694281, Research in Mathematics Education, 14(2), 137-161 (2012)

Hidi, S. y K.A. Renninger, The four phase model of interest development, doi: 10.1207/s15326985ep4102_4, Educational Psychologist, 41(2), 111-127 (2006)

Lobato, C. y A. Madinabeitia, Perfiles Motivacionales de Profesorado ante la Formación en Metodologías Activas en la Universidad, doi: 10.4067/s0718-50062011000100006, Formación Universitaria, 4(1), 137-48 (2011)

Matthews, A.R., C. Hoessler, L. Jonker y D. Stockley, Academic Motivation in Calculus, doi: 10.1080/14926156.2013.758328, Canadian Journal of Science, Mathematics and Technology Education, 13(1), 1-17 (2013)

McLeod, D.B., Research on affect in mathematics education: A reconceptualization, Handbook of research on mathematics teaching and learning 1를 Ed., National Council of Teachers of Mathematics, pp 575-596, Virginia, Estados Unidos (1992)

Morgan, D.L., R.A. Krueger y A.U. Scannell, Planning focus groups, Vol. 2, Sage,Thousand Oaks, California (1998)

Nguyen, G.T., Teacher knowledge of students and enactment of motivational strategies in teaching the concept of functions, Tesis doctoral, Florida State University, Tallahassee, Florida (2011) 
Oriol-Granado, X., M. Mendoza-Lira, C.G. Covarrubias-Apablaza y V.M. Molina- López, Emociones positivas, apoyo a la autonomía y rendimiento de estudiantes universitarios: el papel mediador del compromiso académico y la autoeficacia, doi: 10.1387/RevPsicodidact.14280, Revista de Psicodidáctica, 22(1), 45-53 (2017)

Rakoczy, K., B. Harks y otros tres autores, Written feedback in mathematics: Mediated by students' perception, moderated by goal orientation, doi: 10.1016/j.learninstruc.2013.03.002, Learning and Instruction, 27, 63-73. (2013)

Ryan, R.M. y E.L. Deci, Overview of self-determination theory: An organismic-dialectical perspective, Handbook of selfdetermination research, 1ㄹ Ed., University of Rochester Press, pp 3-33, Nueva York, Estados Unidos (2002)

Ryan, R.M. y E.L. Deci, Self-determination theory and the facilitation of intrinsic motivation, social development and wellbeing, doi: 10.1037110003-066X.55.1.68, American Psychologist, 55(1), 68-78 (2000)

Ryan, R.M., The Oxford handbook of human motivation, $1^{\text {a }}$ Ed., Oxford University Press, Oxford, Inglaterra (2012)

Schukajlow, S. y A. Krug, Do multiple solutions matter? Prompting multiple solutions, interest, competence, and autonomy, doi: 10.5951/jresematheduc.45.4.0497, Journal for Research in Mathematics Education, 45(4), 497-533 (2014)

Schukajlow, S., Is boredom important for students' performance?, Proceedings of the Ninth Congress of the European Society for Research in Mathematics Education, Faculty of Education Charles University and ERME, 1273-1279, Praga, República Checa (2015)

Schukajlow, S., K. Rakoczy y R. Pekrun, Emotions and motivation in mathematics education: theoretical considerations and empirical contributions, doi: 10.1007/s11858-017-0864-6, ZDM, 49(3), 307-322 (2017)

Soler, P., La investigación cualitativa en marketing y publicidad: el grupo de discusión y el análisis de datos, 1ª Ed., Paidos Papeles de Comunicación, Vol. 12, Paidós Ibérica, Barcelona, España (1997)

Vaismoradi, M., H. Turunen y T. Bondas, Content analysis and thematic analysis: Implications for conducting a qualitative descriptive study, doi: 10.1111/nhs.12048, Nursing and Health Sciences, (15), 398-405 (2013) 